\title{
Association between suicide risk severity and sarcopenia in non-elderly Chinese inpatients with major depressive disorder
}

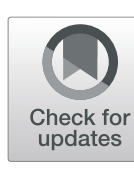

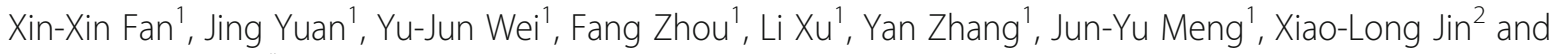
Jian-Zhong Yang ${ }^{1 *}$

\begin{abstract}
Background: Sarcopenia is a skeletal muscle disorder. Recent studies have shown an association between muscle health and suicide. However, there have been no previous studies on the relationship between suicide risk severity and sarcopenia in major depressive disorder (MDD). This study aimed to explore the association between suicide risk severity and sarcopenia in non-elderly Chinese inpatients with MDD.

Methods: The first-episode drug-naïve MDD inpatients aged 20-59 years with the 24-item Hamilton Rating Scale for Depression (HAMD-24) scores of $>20$ were included, who were then classified into low, intermediate, high and very high suicide risk groups according to the Nurses' Global Assessment of Suicide Risk (NGASR). The HAMD-24, the Hamilton Rating Scale for Anxiety (HAMA) and the SARC-F questionnaire were used to assess depression severity, anxiety severity and sarcopenia, respectively. The plasma levels of cortisol and adrenocorticotropic hormone (ACTH) were measured.

Results: A total of 192 MDD inpatients (122 females, 70 males; aged $39.3 \pm 11.7$ years) were included, with 12.5\% meeting criteria for sarcopenia. There were significant differences in gender, HAMD score and prevalence of sarcopenia among the suicide risk groups. Adjusted ordinal regression analysis showed that sarcopenia was significantly associated with more severe suicide risk ( $\mathrm{OR}=2.39,95 \% \mathrm{Cl} 1.02-5.58, p=0.044)$ independent of depression severity.
\end{abstract}

Conclusions: This study revealed that sarcopenia was significantly associated with higher suicide risk in non-elderly Chinese MDD inpatients after adjustment for depression severity. Intervention of sarcopenia might be effective in reducing the risk of suicide in non-elderly MDD patients.

Keywords: Major depressive disorder, Sarcopenia, Suicide risk

\footnotetext{
* Correspondence: jzhyang2004@163.com

'Department of Psychiatry, The Second Affiliated Hospital of Kunming Medical University, 374 Dianmian Av, Kunming 650101, Yunnan, China

Full list of author information is available at the end of the article
}

(c) The Author(s). 2020 Open Access This article is licensed under a Creative Commons Attribution 4.0 International License, which permits use, sharing, adaptation, distribution and reproduction in any medium or format, as long as you give appropriate credit to the original author(s) and the source, provide a link to the Creative Commons licence, and indicate if changes were made. The images or other third party material in this article are included in the article's Creative Commons licence, unless indicated otherwise in a credit line to the material. If material is not included in the article's Creative Commons licence and your intended use is not permitted by statutory regulation or exceeds the permitted use, you will need to obtain permission directly from the copyright holder. To view a copy of this licence, visit http://creativecommons.org/licenses/by/4.0/. The Creative Commons Public Domain Dedication waiver (http://creativecommons.org/publicdomain/zero/1.0/) applies to the data made available in this article, unless otherwise stated in a credit line to the data. 


\section{Background}

Suicide is the major cause of mortality worldwide. WHO reported that over 800,000 people died from suicide each year [1]. In 2018, death registration data for the UK showed that age-specific suicide rates peaked among non-elderly adults aged 20-59 years [2]. The mortality data of the US showed that suicide rates kept increasing from 1999 through 2017, especially among those aged 15-64 years [3]. The situation is even worse in patients with major depressive disorder (MDD) who present high risk of suicide and not surprisingly become the major victim of suicidality. A study showed that MDD was associated with a 20 -fold increased risk of suicide [4]. There is evidence that $15 \%$ of those with MDD in community reported at least one suicide attempt in their lifetime [5], and the lifetime suicide prevalence in MDD patients was about $2-12 \%[6,7]$. Given the serious nature of this public health problem, researches on suicide related factors are warranted, especially among nonelderly adults with MDD.

Recently, muscle health has received increasing attention but still far less than psychosocial factors in mental health. Studies have found the association between muscle health and mental health. There is evidence suggesting associations of low muscular strength with risk of self-harm and suicide in both adolescents [8] and the elderly [9] and with death from suicide in middle-aged men [10]. Sarcopenia, a skeletal muscle disorder, is characterized with generalized decreased muscle mass and/or strength or impaired muscle function [11]. Primary sarcopenia is largely attributable to aging, while secondary sarcopenia can occur earlier in life in association with a range of other conditions $[12,13]$. Recent evidence showed that sarcopenia was positively associated with depressive symptoms assessed by self-rating scales among different populations, such as community-dwelling people and patients with type II diabetes, hemodialysis, end-stage kidney disease and cancer [14-17]. Sarcopenia, suicide and depression share common risk factors, such as chronic low-grade inflammation, brain-derived neurotropic factor (BDNF) dysfunction and dysregulated hypothalamic-pituitary-adrenal (HPA) axis [18-21]. These studies suggest that the three muscle health, depression and suicide - may connect with and overlap each other.

However, there have been few studies of sarcopenia among patients with MDD and no previous studies on the relationship between severity of suicide risk and sarcopenia in MDD.

Early identification of potential risk factors for suicide is crucial for suicide prevention in non-elderly patients with MDD. We hypothesized that MDD patients with sarcopenia comorbidity would present higher suicide risk than those without. Since high suicide rates are a popular problem in the non-elderly, and muscle health is age-related, we only studied non-elderly adults with MDD. In order to minimize the effect of antidepressants and recurrence, we only included first-episode drugnaïve MDD patients. This study aimed to explore if sarcopenia was associated with severity of suicide risk in non-elderly inpatients with MDD after adjustment for depression severity.

\section{Methods \\ Study population}

This was a cross-sectional study targeting first-episode drug-naïve MDD inpatients at the psychiatric department of the Second Affiliated Hospital of Kunming Medical University from January to December 2018. We included patients aged 20-59 years who met the criteria of MDD based on the Diagnostic and Statistical Manual of Mental Disorders, Fourth Edition (DSM-IV) with the total scores of the 24-item Hamilton rating scale for depression (HAMD-24) greater than 20 points. The exclusion criteria were current hyperthyroidism, hypothyroidism, mental retardation, chronic or acute heart or renal failure, hepatic insufficiency, pregnancy, cancer, chronic serious physical disabilities, implanted electrical devices, home oxygen therapy, taking long-term systemic corticosteroids and inability to complete self-administered tests or self-rating scales independently. Demographic information and medical history were collected, including gender, age and years of education. All the doctor-administered clinical assessments were completed by two trained psychiatrists.

\section{Assessment of depression related scales}

The HAMD-24 was used to assess the severity of depression and all the participants in this study scored greater than 20 points [22]. The Hamilton Rating Scale for Anxiety (HAMA) was used to assess the severity of anxiety [23]. The Nurses' Global Assessment of Suicide Risk (NGASR) was used to assess the suicide risk of the participants [24]. It is a risk assessment tool of suicide, incorporating key indicators of suicide based on current evidence regarding suicide. There are 15 items: feelings of hopelessness, recent stressful life events, persecutory voices/beliefs, depression/loss of interest, withdrawal from interpersonal and social interactions, verbalization of suicide intent, a plan to commit suicide, family history of severe psychiatric problems or suicide, recent bereavement or relationship breakdown, personal history of psychosis, widow/widower, prior suicide attempt, history of socio-economic deprivation, history of alcohol and/or alcohol misuse, and presence of a terminal illness. The suicide risk in this manuscript determined by NGASR means the risk of committing suicide. A total score of $\leq 5,6-8,9-11$ and $\geq 12$ points were classified as low, intermediate, high and very high suicide risk, respectively. The Chinese version of NGASR was proved 
to have good reliability and content validity according to Beck Hopelessness Scale (BSS) and Beck Scale for Suicide Ideation (BSI) $[25,26]$. Currently NGASR is widely used in clinical practice and psychiatric researches in inpatients of psychiatric departments [27-30].

\section{Laboratory assays}

Blood samples were collected from the antecubital vein after overnight fasting. Plasma cortisol and adrenocorticotropic hormone $(\mathrm{ACTH})$ levels were measured by chemiluminescence immunoassay (AutoLumo A2000).

\section{Assessment of sarcopenia}

Sarcopenia was evaluated by the SARC-F questionnaire. It is a 5-item mnemonic with each capital letter presenting an item for assessing - Strength, Assistance in walking, Rise from a chair, Climb stairs and Falls. Each item is scored ranging from 0 to 2 points. In 2013, Malmstrom and Morley developed this questionnaire to screen sarcopenia simply, rapidly and conveniently, and suggested that a total score of $\geq 4$ points was considered to indicate sarcopenia [31]. The SARC-F has been validated in different ethnic populations and countries including Chinese, and has been proven valid and consistent for identifying those at risk of sarcopenia in these populations [13], with high specificity but relatively low sensitivity [32-35].

\section{Statistical analyses}

Statistical analyses were performed by SPSS 25.0 (Statistical Package for Social Sciences, SPSS inc). Continuous data were presented as mean \pm standard deviation (SD) if normally distributed, or as median [interquartile range] if skewedly distributed. Categorical data were presented as absolute numbers and percentages. Demographic and clinical characteristics were compared across different severities of suicide risk with the categorical data by $\chi^{2}$ tests or Fisher's exact tests and continuous data by oneway ANOVA or Kruskal-Wallis $\mathrm{H}$ rank sum tests.
Ordinal regression models were then used to evaluate the factors associated with severity of suicide risk. Statistical significance was defined as a two-sided $p$ value of less than 0.05 .

\section{Results}

Of the 245 MDD inpatients who met the inclusion criteria, 25 were excluded and 28 had missing data. The remaining 192 first-episode drug-naïve MDD inpatients (122 female and 70 males; aged $39.3 \pm 11.7$ years) were analyzed in this study.

Among the 192 inpatients included in this study, 69 (35.9\%) were grouped in the low risk group, 76 (39.6\%) in the intermedium risk group, $21(10.9 \%)$ in the high risk group and 26 (13.5\%) in the very high risk group. 24 (12.5\%) inpatients were screened as having sarcopenia, including 4 males (5.7\%) and 20 females (16.4\%), with significant difference in gender $(p=0.040)$. Table 1 summarizes the comparisons of demographic and clinical characteristics by severity of suicide risk. There were significant differences in gender, HAMD score and prevalence of sarcopenia among the suicide risk groups, while no significant differences were found in age, years of education, disease duration, HAMA score, cortisol level and ACTH level.

The results of unadjusted and adjusted ordinal regression models using severities of suicide risk as the dependent variable and other variables as the explanatory variables were shown in Table 2. In the unadjusted model, sarcopenia was significantly associated with severer suicide risk $(\mathrm{OR}=3.85,95 \% \mathrm{CI} 1.73-8.56, p=0.001)$. After adjustment for HAMD score, age and gender, sarcopenia remained significantly associated with severer suicide risk $(\mathrm{OR}=2.39,95 \% \mathrm{CI} 1.02-5.58, p=0.044)$.

\section{Discussion}

The aim of the study was to investigate whether sarcopenia was associated with severity of suicide risk in nonelderly inpatients with MDD after adjustment for

Table 1 Comparison of the characteristics by severity of suicide risk

\begin{tabular}{llllll}
\hline Variables & Low & $\begin{array}{l}\text { Intermedium } \\
N=76\end{array}$ & $\begin{array}{l}\text { High } \\
N=21\end{array}$ & $\begin{array}{l}\text { Very high } \\
N=26\end{array}$ \\
\hline Age (years) & $40.7 \pm 11.4$ & $40.1 \pm 11.2$ & $39.0 \pm 13.0$ & $33.9 \pm 11.9$ & 0.075 \\
Gender (Female) & $39(56.5 \%)$ & $45(59.2 \%)$ & $15(71.4 \%)$ & $23(63.5 \%)$ & $13.2 \pm 3.1$ \\
Education (years) & $12.8 \pm 4.2$ & $12.8 \pm 3.9$ & $11.5 \pm 5.1$ & $21.08 \pm 8.57$ & $0.004^{*}$ \\
Cortisol $(\mu \mathrm{g} / \mathrm{dL})$ & $19.33 \pm 8.01$ & $21.46 \pm 10.52$ & $21.88 \pm 8.41$ & $32.05[30.86]$ & 0.487 \\
ACTH $(\mathrm{pg} / \mathrm{mL})$ & $32.54[36.87]$ & $32.79[32.65]$ & $39.23[34.11]$ & $38.9 \pm 6.1$ & 0.520 \\
HAMD score & $26.0 \pm 4.7$ & $30.3 \pm 7.9$ & $36.8 \pm 6.2$ & $19.2 \pm 9.7$ & $<0.001^{* *}$ \\
HAMA score & $16.6 \pm 7.5$ & $17.0 \pm 8.7$ & $20.5 \pm 8.3$ & $6(23.1 \%)$ & 0.187 \\
Sarcopenia & $2(2.9 \%)$ & $10(13.2 \%)$ & $6(28.6 \%)$ & $0.001^{*}$ \\
\hline
\end{tabular}

Data are presented as mean $\pm \mathrm{SD}$, median [interquartile range] or absolute numbers (percentage)

${ }^{*} p<0.05$; ${ }^{* *} p<0.001$ 
Table 2 Association of suicide risk with sarcopenia in unadjusted and adjusted ordinal regression models

\begin{tabular}{|c|c|c|c|c|c|c|}
\hline Variables & $\begin{array}{l}\text { Unadjusted } \\
\text { OR (95\% Cl) }\end{array}$ & $P$ & $\begin{array}{l}\text { Model } 1 \\
\text { OR (95\% Cl) }\end{array}$ & P & $\begin{array}{l}\text { Model } 2 \\
\text { OR (95\% Cl) }\end{array}$ & $P$ \\
\hline Sarcopenia & $\begin{array}{c}3.85 \\
(1.73-8.56)\end{array}$ & $0.001^{*}$ & $\begin{array}{l}2.36 \\
(1.01-5.50)\end{array}$ & $0.047^{*}$ & $\begin{array}{l}2.39 \\
(1.02-5.58)\end{array}$ & $0.044^{*}$ \\
\hline HAMD score & & & $\begin{array}{l}1.16 \\
(1.12-1.21)\end{array}$ & $<0.001^{* *}$ & $\begin{array}{l}1.16 \\
(1.11-1.21)\end{array}$ & $<0.001^{* *}$ \\
\hline Male & & & $\begin{array}{l}0.74 \\
(0.41-1.33)\end{array}$ & 0.311 & $\begin{array}{l}0.73 \\
(0.40-1.31)\end{array}$ & 0.288 \\
\hline Age & & & & & $\begin{array}{l}0.99 \\
(0.97-1.02)\end{array}$ & 0.621 \\
\hline
\end{tabular}

Model 1: adjusted for HAMD score and gender

Model 2: adjusted for HAMD score, gender and age

$\mathrm{OR}=$ odds of being in a more severe suicide risk group

${ }^{*} p<0.05$; ** $p<0.001$

depression severity. The results suggest that those with severer depression and sarcopenia comorbidity have higher suicide risk, and sarcopenia is significantly associated with severity of suicide risk even in the significant context of depression severity, which indicate that sarcopenia screening and identification is essential in MDD patients.

Our study also showed that in non-elderly MDD patients, females tend to have higher suicide risk than males, consistent with some previous studies [36]. While another study found males did more suicides than females [37], which may attribute to the difference in suicide assessments. As to the relationship between HPA axis and suicide risk, it has been suggested that stressful psychological events may be used to predict suicide risk [38], and the function of HPA axis has been reported to be associated with suicide through regulating the response to stress, but the results are inconsistent [39-42]. Our study found no significant differences in ACTH and cortisol levels across suicide risk severity groups. So prospective studies with large sample and consistent study design may be needed to explore the relationship.

Previous studies have reported associations between suicide and muscle-related indicators. Specifically, suicide was found positively associated with decreased skeletal muscle strength $[8-10,43]$, poorer physical performance [9], sarcopenia [44, 45] and physical inactivity $[46,47]$. In line with these researches, our study found that sarcopenia, a skeletal muscle disorder, was associated with higher suicide risk. Therefore, mechanisms of the relationship between sarcopenia and suicide deserve further investigation.

Current researches suggest some underlying mechanisms for the association between suicide and muscle. From the perspective of psychosocial factors, decreased muscle strength was associated with lowered selfconcept and self-esteem $[48,49]$ that could increase suicide risk, while physical exercise may both benefit those with sarcopenia and those with high suicide risk $[46,50]$, from which we could infer that sarcopenia is associated with suicide, consistent with our result.

The association might also be explained from the perspective of biological factors. A study on biomarkers of suicide by Niculescu et al. have shown that patients with higher suicide risk have relatively high levels of interleukin6 (IL-6) [21] that involved in inflammation response. While chronic low-grade inflammation might increase muscle catabolism, which is one of the pathophysiological characteristics of sarcopenia [51]. Further, these pro-inflammatory cytokines can activate kynurenine pathway [52-54], increase the level of quinolinic acid, the NMDA receptor agonist, and simultaneously decrease neuroprotective metabolites via glutamate neurotransmission and neuroinflammation [55]. Whereas physical exercise induces the release of PGC-1 1 in skeletal muscle that increases the expression of kynurenine aminotransferases, thus promoting the conversion of kynurenine into kynurenic acid, a metabolite unable to pass the blood-brain barrier, and protecting the brain from stress-induced changes, which can be instrumental in relieving depression and suicide ideation [56]. Moreover, BDNF, a key regulator of brain development and plasticity, is related to suicide risk when it is dysfunctional [19]. While physical exercise mediates central BDNF production in humans [57], and have positive effects on brain plasticity and increases brain reserve capacity, which might reduce suicide risk [58, 59]. In brief, the relationship between sarcopenia and suicide may be linked by inflammation, kynurenine metabolism and brain plasticity, and potential intervention in these factors might not only relieve depression and suicide ideation, but also improve sarcopenia, as our results suggest.

\section{Strengths and limitations}

To the best of our knowledge, this was the first study to reveal that sarcopenia, screened by the recently developed simple SARC-F questionnaire, was associated with suicide risk severity in non-elderly fist-episode drugnaïve MDD inpatients. Early identifying and intervention 
for sarcopenia might ameliorate suicide risk in MDD patients.

There existed a number of limitations in this study. First, the SARC-F questionnaire was used as the way of identifying sarcopenia in this study. However, SARC-F was a simple screening tool rather than a diagnostic criterion for sarcopenia, with relatively high specificity and low-to-moderate sensitivity, which might affect the validity of this study. Accordingly, those identified as sarcopenia should have further test for body composition in order to make a definite diagnosis. Second, more females and the small sample size of sarcopenia in this study might lead to biased results and limit the generality and explanatory power. Additionally, as this was a crosssectional study, the causal relationship between sarcopenia and suicide risk was unable to determine. Whether sarcopenia could affect the efficacy of antidepressant therapy or worsen the prognosis is worth further studying. Therefore, future prospective studies with larger samples should be conducted among MDD patients with comorbid sarcopenia at different ages to explore whether there will be therapeutic response and brain changes together with or dependent of depression treatment after intervention of sarcopenia.

\section{Conclusions}

This study revealed that sarcopenia was significantly associated with higher suicide risk in non-elderly MDD inpatients after adjustment for depression severity. Educating psychiatric staffs about sarcopenia screening, identifying and intervening is important. Improving the patients' physical fitness might help MDD patients to reduce suicide risk and get better social rehabilitation. Future prospective researches are needed to elucidate whether suicide risk could be reduced through intervention of sarcopenia.

\section{Abbreviations}

ACTH: Adrenocorticotropic hormone; BDNF: Brain-derived neurotropic factor: BSI: Beck Scale for Suicide Ideation; BSS: Beck Hopelessness Scale; DSMIV: Diagnostic and Statistical Manual of Mental Disorders, Fourth Edition; HAMD-24: 24-item Hamilton rating scale for depression; HAMA: Hamilton Rating Scale for Anxiety; HPA: Hypothalamic-pituitary-adrenal; IL6: Interleukin-6; MDD: Major depressive disorder; NGASR: 15-item Nurses' Global Assessment of Suicide Risk; SD: Standard deviation

\section{Acknowledgements}

The authors acknowledge the efforts of this research team and appreciate all the participants for attending this research.

\section{Authors' contributions}

$X X F$ and JZY designed the research. XXF, JZY and XLJ wrote the manuscript and analyzed the data. JY, YJW, LX, YZ, FZ and JYM contributed to the data collection. Special thanks to Prof. Gavin Reynolds for assistance in writing the paper. All authors have read and approved the manuscript.

\section{Funding}

This research was supported by the National Key R\&D Program of China (JZY, grant numbers 2018YFC 1314400, 2018YFC 1314405); the National Natural Scientific Foundation of China (JZY, grant numbers 81160170 and 81660235); the training program for leading personnel of Yunnan Health Planning Committee (JZY, grant number L-201623); the young and middle-aged academic and technical leaders in Yunnan Province, China (JZY, grant number 2018HB017); and the scientific research foundation of education department in Yunnan province, China (XXF, grant number 2019Y0359).

\section{Availability of data and materials}

The data used in the current study are available from the corresponding author for reasonable request.

\section{Ethics approval and consent to participate}

This study was carried out in accordance with the Declaration of Helsinki as revised 1989, and approved by the Ethics Committee of the Second Affiliated Hospital of Kunming Medical University. All participants provided written and informed consent.

\section{Consent for publication}

Not applicable.

\section{Competing interests}

The authors declare no competing interests.

\section{Author details}

${ }^{1}$ Department of Psychiatry, The Second Affiliated Hospital of Kunming Medical University, 374 Dianmian Av, Kunming 650101, Yunnan, China.

2Department of Geriatrics, The Sixth Affiliated Hospital of Kunming Medical University (People's Hospital of Yuxi), Yuxi, Yunnan, China.

Received: 8 March 2020 Accepted: 25 June 2020

Published online: 02 July 2020

\section{References}

1. WHO. Preventing suicide: a resource for media professionals, update 2017 Geneva: World Health Organization; 2017. (WHO/MSD/MER/17.5). 2017.

2. Office for National Statistics. Suicides in the UK: 2018 registrations, https:// www.ons.gov.uk/peoplepopulationandcommunity/ birthsdeathsandmarriages/deaths/bulletins/suicidesintheunitedkingdom/201 8registrations; 2019 [accessed 3 September 2019].

3. Hedegaard H, Curtin SC, Warner M. Suicide Mortality in the United States, 1999-2017. NCHS Data Brief 2018(330):1-8.

4. Osby U, Brandt L, Correia N, Ekbom A, Sparen P. Excess mortality in bipolar and unipolar disorder in Sweden. Arch Gen Psychiatry. 2001;58(9):844-50.

5. Chen YW, Dilsaver SC. Lifetime rates of suicide attempts among subjects with bipolar and unipolar disorders relative to subjects with other Axis I disorders. Biol Psychiatry. 1996;39(10):896-9.

6. Bostwick JM, Pankratz VS. Affective disorders and suicide risk: a reexamination. Am J Psychiatry. 2000;157(12):1925-32.

7. Sinclair JM, Harriss L, Baldwin DS, King EA. Suicide in depressive disorders: a retrospective case-control study of 127 suicides. J Affect Disord. 2005;87(1): 107-13.

8. Ortega FB, Silventoinen K, Tynelius P, Rasmussen F. Muscular strength in male adolescents and premature death: cohort study of one million participants. BMJ. 2012;345:e7279.

9. Bickford D, Morin RT, Woodworth C, Verduzco E, Khan M, Burns E, et al. The relationship of frailty and disability with suicidal ideation in late life depression. Aging Ment Health. 2019:1-6.

10. Nyberg J, Gustavsson S, Aberg MAl, Kuhn HG, Waern M. Late-adolescent risk factors for suicide and self-harm in middle-aged men: explorative prospective population-based study. Br J Psychiatry. 2019:1-7.

11. Fielding RA, Vellas B, Evans WJ, Bhasin S, Morley JE, Newman AB, et al. Sarcopenia: an undiagnosed condition in older adults. Current consensus definition: prevalence, etiology, and consequences. International working group on sarcopenia. J Am Med Dir Assoc. 2011;12(4):249-56.

12. Cruz-Jentoft AJ, Sayer AA. Sarcopenia Lancet. 2019;393(10191):2636-46.

13. Cruz-Jentoft AJ, Bahat G, Bauer J, Boirie Y, Bruyere O, Cederholm T, et al. Sarcopenia: revised European consensus on definition and diagnosis. Age Ageing. 2019;48(1):16-31

14. Ida S, Murata K, Nakai M, Ito S, Malmstrom TK, Ishihara Y, et al. Relationship between sarcopenia and depression in older patients with diabetes: an investigation using the Japanese version of SARC-F. Geriatr Gerontol Int 2018;18(9):1318-22. 
15. Alston H, Burns A, Davenport A. Loss of appendicular muscle mass in haemodialysis patients is associated with increased self-reported depression, anxiety and lower general health scores. Nephrology (Carlton). 2018;23(6): 546-51.

16. Kim JK, Choi SR, Choi MJ, Kim SG, Lee YK, Noh JW, et al. Prevalence of and factors associated with sarcopenia in elderly patients with end-stage renal disease. Clin Nutr. 2014;33(1):64-8.

17. Nipp RD, Fuchs G, El-Jawahri A, Mario J, Troschel FM, Greer JA, et al. Sarcopenia is associated with quality of life and depression in patients with advanced Cancer. Oncologist. 2018;23(1):97-104.

18. Budui SL, Rossi AP, Zamboni M. The pathogenetic bases of sarcopenia. Clin Cases Miner Bone Metab. 2015;12(1):22-6.

19. Dwivedi Y. Brain-derived neurotrophic factor and suicide pathogenesis. Ann Med. 2010;42(2):87-96.

20. O'Connor DB, Branley-Bell D, Green JA, Ferguson E, O'Carroll RE, O'Connor RC. Effects of childhood trauma, daily stress, and emotions on daily cortisol levels in individuals vulnerable to suicide. J Abnorm Psychol. 2020;129(1):92-107.

21. Niculescu AB, Levey DF, Phalen PL, Le-Niculescu H, Dainton HD, Jain N, et al. Understanding and predicting suicidality using a combined genomic and clinical risk assessment approach. Mol Psychiatry. 2015;20(11):1266-85.

22. Hamilton M. Development of a psychiatric rating scale for primary depression. Brit Soc Clin Psychol. 1967;6:278-96.

23. Hamilton M. The assessment of anxiety by rating scale. Brit J Med Psychol. 1959;32:50-5.

24. Cutcliffe JR, Barker P. The Nurses' global assessment of suicide risk (NGASR): developing a tool for clinical practice. J Psychiatr Ment Health Nurs. 2004; 11(4):393-400.

25. Chen Y, Ye M, Ji X, Fang C, Chen Y. Validity and reliability of Nurses' global assessment of suicide risk (NGASR) for schizophrenia inpatients. Med $J$ Chin People's Health. 2011;3.

26. Guo J, Lu Q, Chen X, Liu F. Reliability and validity of the retranslated China version of Nurses' global assessment of suicide risk scale. Chin J Pract Nursing. 2018;24:1841-5.

27. Li X, Ge H, Zhou D, Wu X, Qi G, Chen Z, et al. Reduced serum VGF levels are linked with suicide risk in Chinese Han patients with major depressive disorder. BMC Psychiatry. 2020;20(1):225

28. Gramaglia C, Feggi A, Bergamasco P, Bert F, Gattoni E, Marangon D, et al. Clinical characteristics associated with suicide attempts in clinical settings: a comparison of suicidal and non-suicidal depressed inpatients. Front Psychiatry. 2016;7:109.

29. Yang Y, Chattun MR, Yan R, Zhao K, Chen Y. Zhu R, et al. Brain Imaging Behav: Atrophy of right inferior frontal orbital gyrus and frontoparietal functional connectivity abnormality in depressed suicide attempters; 2020.

30. Chattun MR, Zhang S, Chen Y, Wang Q, Amdanee N, Tian S, et al. Caudothalamic dysfunction in drug-free suicidally depressed patients: an MEG study. Eur Arch Psychiatry Clin Neurosci. 2020;270(2):217-27.

31. Malmstrom TK, Morley JE. SARC-F: a simple questionnaire to rapidly diagnose sarcopenia. J Am Med Dir Assoc. 2013;14(8):531-2.

32. Parra-Rodriguez L, Szlejf C, Garcia-Gonzalez Al, Malmstrom TK, Cruz-Arenas E, Rosas-Carrasco O. Cross-cultural adaptation and validation of the Spanishlanguage version of the SARC-F to assess sarcopenia in Mexican community-dwelling older adults. J Am Med Dir Assoc. 2016;17(12):1142-6.

33. Kim S, Kim M, Won CW. Validation of the Korean version of the SARC-F questionnaire to assess sarcopenia: Korean frailty and aging cohort study. J Am Med Dir Assoc. 2018:19(1):40-5 e1.

34. Kemmler W, Sieber C, Freiberger E, von Stengel S. The SARC-F questionnaire: diagnostic overlap with established sarcopenia definitions in older German men with sarcopenia. Gerontology. 2017;63(5):411-6.

35. Cao L, Chen S, Zou C, Ding X, Gao L, Liao Z, et al. A pilot study of the SARCF scale on screening sarcopenia and physical disability in the Chinese older people. J Nutr Health Aging. 2014;18(3):277-83.

36. Dendup T, Zhao Y, Dorji T, Phuntsho S. Risk factors associated with suicidal ideation and suicide attempts in Bhutan: an analysis of the 2014 Bhutan STEPS survey data. PLoS One. 2020;15(1):e0225888.

37. Cuchara B, Diaz FJ. An 8-year retrospective study on suicides in Washington. DC Am J Forensic Med Pathol. 2020;41(1):18-26.

38. van Heeringen K, Mann JJ. The neurobiology of suicide. Lancet Psychiatry. 2014;1(1):63-72.

39. O'Connor DB, Ferguson E, Green JA, O'Carroll RE, O'Connor RC. Cortisol levels and suicidal behavior: a meta-analysis. Psychoneuroendocrinology. 2016:63:370-9.
40. Papadopoulou A, Douzenis A, Christodoulou C, Gournellis R, Papageorgiou C, Markianos M. Association of Plasma Cortisol Levels with clinical characteristics of suicide attempters. Neuropsychobiology. 2017;76(3):161-5.

41. Kim B, Kang ES, Fava M, Mischoulon D, Soskin D, Yu BH, et al. Folliclestimulating hormone (FSH), current suicidal ideation and attempt in female patients with major depressive disorder. Psychiatry Res. 2013;210(3):951-6.

42. Pompili M, Serafini G, Innamorati M, Moller-Leimkuhler AM, Giupponi G, Girardi $P$, et al. The hypothalamic-pituitary-adrenal axis and serotonin abnormalities: a selective overview for the implications of suicide prevention. Eur Arch Psychiatry Clin Neurosci. 2010;260(8):583-600.

43. Lee K. Relative handgrip strength in relation to depressive mood and suicidal ideation in Koreans using the 2015 KNHANES data. J Musculoskelet Neuronal Interact. 2018;18(3):333-8.

44. Kim JH, Kim DH, Park YS. Body composition, sarcopenia, and suicidal ideation in elderly Koreans: Hallym aging study. J Korean Med Sci. 2016; 31(4):604-10.

45. Cho Y, Shin S-Y, Shin M-J. Sarcopenic obesity is associated with lower indicators of psychological health and quality of life in Koreans. Nutr Res (New York, NY). 2015;(5):384-92.

46. Davidson CL, Babson KA, Bonn-Miller MO, Souter T, Vannoy S. The impact of exercise on suicide risk: examining pathways through depression, PTSD, and sleep in an inpatient sample of veterans. Suicide Life Threat Behav. 2013; 43(3):279-89.

47. Sturm J, Ploderl M, Fartacek C, Kralovec K, Neunhauserer D, Niederseer D, et al. Physical exercise through mountain hiking in high-risk suicide patients. A randomized crossover trial. Acta Psychiatr Scand. 2012;126(6):467-75.

48. Ciccolo JT, SantaBarbara NJ, Dunsiger SI, Busch AM, Bartholomew JB. Muscular strength is associated with self-esteem in college men but not women. J Health Psychol. 2016;21(12):3072-8.

49. Greenleaf CA, Petrie TA, Martin SB. Psychosocial variables associated with body composition and cardiorespiratory fitness in middle school students. Res Q Exerc Sport. 2010;81(3 Suppl):S65-74.

50. Lee SY, Tung HH, Liu CY, Chen LK. Physical activity and sarcopenia in the geriatric population: a systematic review. J Am Med Dir Assoc. 2018;19(5):378-83.

51. Bano G, Trevisan C, Carraro S, Solmi M, Luchini C, Stubbs B, et al. Inflammation and sarcopenia: a systematic review and meta-analysis. Maturitas. 2017:96:10-5.

52. Bryleva EY, Brundin L. Kynurenine pathway metabolites and suicidality Neuropharmacology. 2017;112(Pt B):324-30.

53. Schwieler L, Larsson MK, Skogh E, Kegel ME, Orhan F, Abdelmoaty S, et al. Increased levels of IL-6 in the cerebrospinal fluid of patients with chronic schizophrenia--significance for activation of the kynurenine pathway. J Psychiatry Neurosci. 2015;40(2):126-33.

54. Urata Y, Koga K, Hirota Y, Akiyama I, Izumi G, Takamura M, et al. IL-1 beta increases expression of tryptophan 2,3-dioxygenase and stimulates tryptophan catabolism in endometrioma stromal cells. Am J Reprod Immunol. 2014;72(5):496-503.

55. Kaiser H, Yu K, Pandya C, Mendhe B, Isales CM, McGee-Lawrence ME, et al. Kynurenine, a tryptophan metabolite that increases with age, Induces Muscle Atrophy and Lipid Peroxidation. Oxid Med Cell Longev. 2019;2019: 9894238.

56. Agudelo LZ, Femenia T, Orhan F, Porsmyr-Palmertz M, Goiny M, MartinezRedondo $V$, et al. Skeletal muscle PGC-1alpha1 modulates kynurenine metabolism and mediates resilience to stress-induced depression. Cell. 2014; 159(1):33-45.

57. Pedersen BK. Physical activity and muscle-brain crosstalk. Nat Rev Endocrinol. 2019;15(7):383-92.

58. Nithianantharajah J, Hannan AJ. The neurobiology of brain and cognitive reserve: mental and physical activity as modulators of brain disorders. Prog Neurobiol. 2009;89(4):369-82.

59. Delezie J, Handschin C. Endocrine crosstalk between skeletal muscle and the brain. Front Neurol. 2018;9:698

\section{Publisher's Note}

Springer Nature remains neutral with regard to jurisdictional claims in published maps and institutional affiliations. 